\title{
Tetramethylpyrazine Inhibits Activation of Hepatic Stellate Cells through Hedgehog Signaling Pathways In Vitro
}

\author{
Jue Hu, ${ }^{1}$ Gang Cao, ${ }^{2,3} \mathrm{Xin} \mathrm{Wu}^{2}{ }^{\mathrm{Hao}} \mathrm{Cai}^{3}$ and Baochang Cai ${ }^{3}$ \\ ${ }^{1}$ Zhejiang Medical College, Hangzhou 310053, China \\ ${ }^{2}$ Research Center of TCM Processing Technology, Zhejiang Chinese Medical University, Hangzhou 311401, China \\ ${ }^{3}$ Department of Chinese Materia Medica, College of Pharmacy, Nanjing University of Chinese Medicine, Nanjing 210023, China
}

Correspondence should be addressed to Gang Cao; caogang33@163.com

Received 12 May 2015; Accepted 12 August 2015

Academic Editor: Swaran J. S. Flora

Copyright (C) 2015 Jue Hu et al. This is an open access article distributed under the Creative Commons Attribution License, which permits unrestricted use, distribution, and reproduction in any medium, provided the original work is properly cited.

\begin{abstract}
Background and Aim. Tetramethylpyrazine (TMP), a major alkaloid isolated from Ligusticum chuanxiong, has been reported in hepatic fibrosis models. However, the action mechanism remains unclear. In the present study, effects of tetramethylpyrazine (TMP) against hepatic stellate cell (HSC) activation as well as the possible mechanisms were evaluated. Methods. Western blot assay was used to detect TMP effects on protein expression of Smo, Patched, Hhip, and Gli and to investigate the effects of TMP on Cyclin D1, Cyclin E1, CDK2, Bcl-2, Bax, and caspase expression with cyclopamine supplementation. Results. Our results showed that TMP significantly inhibits the expression of Cyclin D1, Cyclin E1, and Cyclin-dependent kinase CDK2 and changes the HSC cycle by inhibiting the proliferation of HSC. Moreover, TMP has also been shown to decrease the expression of Bcl-2 and increase the expression of Bax in HSC-T6 cells. Furthermore, TMP can inhibit the expression of connective tissue growth factor (CTGF), and the inhibitory effect was intensified after the application of joint treatment with TMP and cyclopamine. Conclusion. TMP may be an effective Hh signaling pathway inhibitor for hepatic fibrosis treatment.
\end{abstract}

\section{Introduction}

Hepatic fibrosis is the compensatory reaction during the process of tissue repair secondary to various forms of chronic liver damage (related to alcohol, viral infection, or drugs) $[1,2]$. In the absence of effective therapeutic measures, continued development of the disease will alter the normal liver structure and hepatic function. The liver will also develop hepatic cirrhosis because of hepatic insufficiency. Ultimately, this condition may lead to hepatocellular carcinoma. In the development process of hepatic fibrosis, activation, the proliferation of HSC provides key contributions. Therefore, the activation, proliferation, and apoptosis of HSC should be investigated to further explore the pathogenesis of hepatic fibrosis. Recent studies indicated that the hedgehog $(\mathrm{Hh})$ signaling pathway may be involved in HSC activation [3, 4], which consequently affect the development of hepatic fibrosis by influencing the process that transforms HSC into myofibroblasts $[5,6]$.
In recent years, increasing number of studies reported about TMP in liver protection and the effects of hepatic fibrosis inhibition [7-10], which showed a promising research topic. In vivo and in vitro studies indicated that TMP can regulate the HSC cycle, thus inhibiting the proliferation of HSC or facilitating the apoptosis of HSC, as well as reducing the expression and sedimentation of extracellular matrix (ECM) [11]. TMP seemed to play the role of antifibrosis, but the action mechanism remains unclear. Although the antihepatic fibrosis effect of TMP has been investigated [12], the report did not explore whether the action mechanism was associated with the regulation of the Hh signaling pathway.

\section{Material and Methods}

2.1. Cell Culture and Treatment. Rat HSC-T6 cell line was purchased from the Type Culture Collection of the Chinese Academy of Sciences (Shanghai, China). The cells were cultured in DMEM medium supplemented with $10 \%$ fetal calf 
serum, $100 \mathrm{U} / \mathrm{mL}$ penicillin, and streptomycin. HSC-T6 cells were incubated at $37^{\circ} \mathrm{C}$ in a $5 \% \mathrm{CO}_{2}$ humidified atmosphere. Culture medium was changed every 2-3 days, and HSC-T6 cells were digested with $0.25 \%$ trypsin and seeded onto a $75 \%$ confluent monolayer.

2.2. Cell Viability. Rat HSC-T6 cells were cultured in 96-well tissue culture plates by adding $180 \mu \mathrm{L} /$ well of a suspension of $1 \times 10^{4}$ cells/well. After cells reached confluence, the medium was replaced with fresh medium supplemented with $10 \%$ fetal calf serum and different doses of TMP and cyclopamine. Following treatment, cell viability was determined by the use of the 3-(4,5-dimethylthiazol-2-yl)-2,5-diphenyltetrazolium bromide (MTT, Sigma) assay and the absorbance at the wavelength of $490 \mathrm{~nm}$ was determined using an automatic microplate reader.

2.3. Western Blot Analysis. The harvested rat HSC-T6 cells were lysed in ice-cold radioimmunoprecipitation (RIPA) buffer. Cell lysates were collected and centrifuged at 12,000 $\times \mathrm{g}$ for $15 \mathrm{~min}$ at $4^{\circ} \mathrm{C}$. Protein concentrations were determined using a bicinchoninic acid (BCA) protein assay kit (Thermo Pierce, USA). Proteins were separated using 12\% SDSPAGE and transferred to a nitrocellulose membranes after electrophoresis. The transferred membranes were incubated with anti-CTGF, anti- $\alpha$ (I) collagen, antifibronectin, anti$\alpha$-SMA, anti-Smo, anti-Patched, anti-Hhip, anti-Gli, antiCyclin D1, anti-Cyclin E1 (CST), anti-CDK2, anti-Bcl-2, antiBax, anti-PARP-1, antiprocaspase-3, antiprocaspase-9, and antiprocaspase-8. Membranes were washed and then incubated with a 1:5000 dilution of secondary antibody for $1 \mathrm{~h}$, and the membranes were detected with the enhanced chemiluminescence system (Amersham Life Science). Proteins were visualized with ECL-chemiluminescent kit (ECL-plus, Thermo Scientific) and normalized to the signal intensity of $\beta$-actin.

2.4. Statistical Analysis. All results are expressed as the mean \pm SD. Statistical significance was determined by the Student's $t$-test, and the level of $p<0.05$ was taken as significant difference.

\section{Results}

3.1. TMP Inhibits Both Viability and Activation of HSC-T6 Cells. HSC-T6 cells were treated with different concentrations of TMP $(10-80 \mu \mathrm{M})$ and durations $(0-48 \mathrm{~h})$ using the MTT assay. TMP-treatment dose dependently reduces HSCT6 cell proliferation slightly at concentrations as low as $30 \mu \mathrm{M}$. Concentrations in the $40-80 \mu \mathrm{M}$ range significantly reduced cell viability; however, cell toxicity of TMP significantly increased. Then, we chose $30 \mu \mathrm{M}$ TMP to intervene in the cells in further experiments. A TMP time-response curve was created, and $12 \mathrm{~h}$ of TMP was found to be the optimum time, which was used in all of the experiments. As shown in Figure 1, western blot analysis for Smo, Patched, and Hhip protein levels showed inhibition of $\mathrm{Hh}$ signaling pathway with a dose-response pattern.
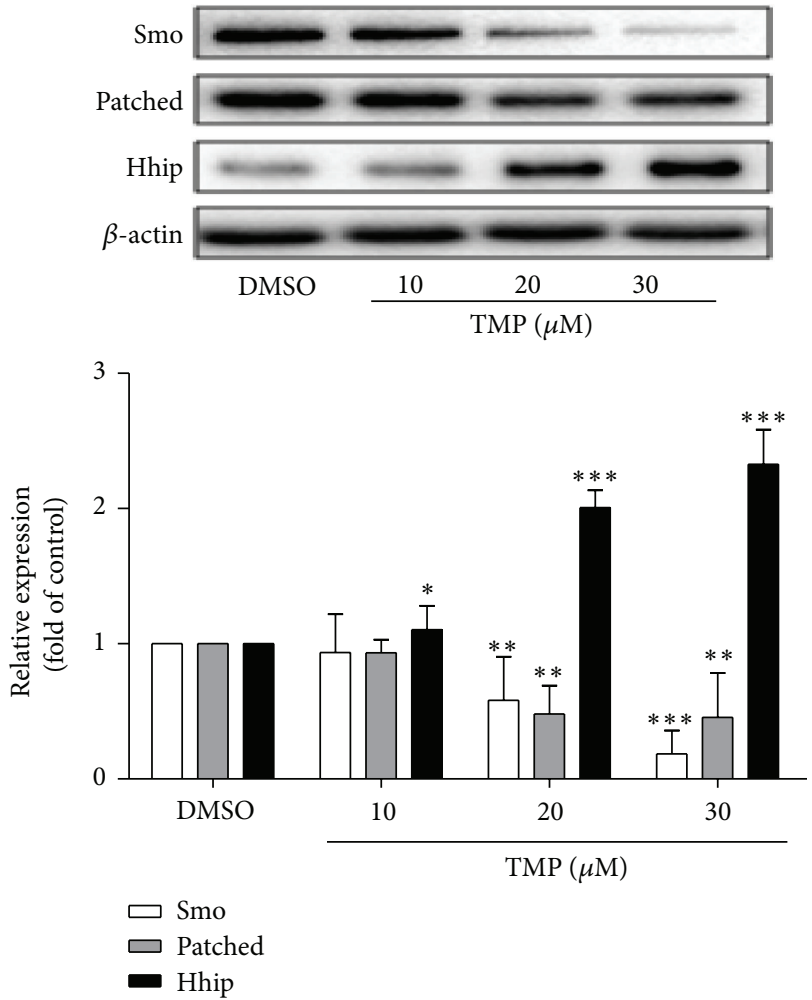

FIGURE 1: TMP inhibits both the activation of HSCs. Effects of TMP on the activities of Smo, Patched and Hhipin HSC-T6 cells. The levels of Smo, Patched, and Hhip protein levels were determined by western blot analysis and normalized by $\beta$-actin. Significance is defined as follows: ${ }^{*} p<0.05,{ }^{* *} p<0.01$, and ${ }^{* *} p<0.001$ compared with control. The DMSO was defined as control.

3.2. TMP Decreases Cyclin D1, Cyclin E1, and CDK2 Activities in HSC-T6 Cells. Liver fibrosis is associated with the proliferation of HSCs, and the cell cycle of activated HSCs is abnormal. Cyclin D1, Cyclin E1, and CDK2 play essential roles in cell proliferation. Thus, the question is whether Cyclin D1, Cyclin E1, and CDK2 activities are inhibited by TMP treatment. Western blot analysis showed that TMP $(30 \mu \mathrm{M})$ and cyclopamine $(10 \mu \mathrm{M}$, specific inhibitor for Hh signaling pathways) significantly reduced the Cyclin D1, Cyclin E1, and CDK2 protein expression (Figure 2). The results showed that TMP significantly induced cell cycle arrest by blocking $\mathrm{Hh}$ signaling pathways.

3.3. TMP Induces HSC-T6 Cell Apoptosis. Caspases have been recognized as important mediators of apoptosis through the cleavage of various cellular substrates. Thus, we assess the effects of TMP on caspase activation by western blotting analyses. The results are as shown in Figure 3; TMP and cyclopamine treatment induced HSC-T6 cell apoptosis by activating caspase-3, PARP-1, caspase-8, and caspase-9. Furthermore, TMP and cyclopamine have synergistic effects on the apoptosis of HSC-T6 cells.

Bcl-2 and Bax, members of the Bcl-2 family of proteins, are antiapoptotic and proapoptotic factors, respectively. Therefore, we investigated whether the ratio of $\mathrm{Bcl}-2 / \mathrm{Bax}$ is 

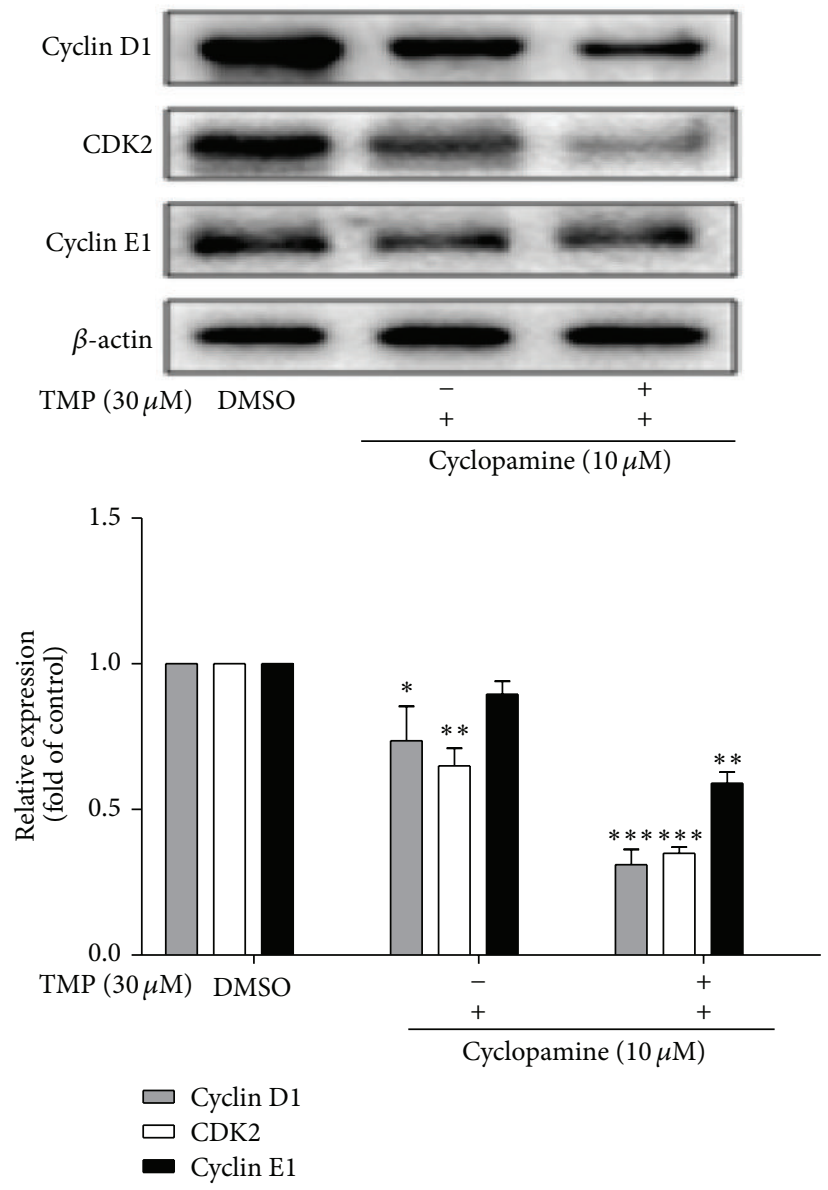

FIGURE 2: Effects of TMP on the activities of Cyclin D1, Cyclin E1, and CDK2 in HSC-T6 cells. Data were reported as means \pm SD. For statistical analysis, ${ }^{*} p<0.05,{ }^{* *} p<0.01$, and ${ }^{* *} p<0.001$ represent significant difference.

inhibited by the pretreatment of cells with TMP. The results showed that TMP has also been shown to decrease the expression of $\mathrm{Bcl}-2$ and increase the expression of Bax in HSC-T6 cells.

3.4. TMP Alters the Expression of Some Fibrotic Marker Proteins in HSC Activation. $\alpha$-SMA is the primary marker of HSC activation, whereas $\alpha 1$ (I) collagen and fibronectin are the major components of ECM secreted by HSC. Western blot assay results indicated that TMP can inhibit the expression of $\alpha$-SMA, $\alpha 1$ (I) collagen, and fibronectin. The expression of CTGF, which mediates development and secretion of ECM and plays a key role in HF development, was found to increase HSC activation (Figure 4). In summary, this study found that TMP can inhibit the expression of CTGF, and the inhibitory effect was intensified after the application of joint treatment with TMP and cyclopamine.

\section{Discussion}

The Hh signal response primarily relies on the control of membrane proteins Ptc and Smo $[13,14]$. The study showed that the expression of both proteins can be significantly inhibited by TMP in a dose-dependent manner. Therefore, the Hh signaling pathway may be the key hub to inhibit HSC activation. In the experiment, TMP showed the potential to inhibit the activities of Cyclin D1, Cyclin E1, and Cyclindependent kinase CDK2 and to change the HSC cycle by inhibiting the proliferation of HSC. Cell cycle arrest was also associated with HSC apoptosis, which is very important to maintain the integrity of the organism and selectively remove excessive or damaged cells. In the report, the treatment of HSC by TMP showed that the ratio of $\mathrm{Bcl}-2 / \mathrm{Bax}$ was downregulated, whereas caspase family proteins were activated. These results indicated that HSC apoptosis can be induced by TMP. Furthermore, TMP can reduce the secretion of $\alpha 1$ (I) procollagen, fibronectin, and CTGF in ECM. The TMP can then stop and reverse the development of HF by inhibiting the HSC activity. Synergistic effects were presented after TMP combined with Hh signaling pathway, and inhibitor cyclopamine was applied.

TMP can improve hepatic fibrosis; however, TMP may exert an antihepatic fibrosis effect by blocking the Hh signaling pathway at G0/G1 phase in cell cycle arrest and triggering caspase-dependent HSC apoptosis. Recent studies reported 

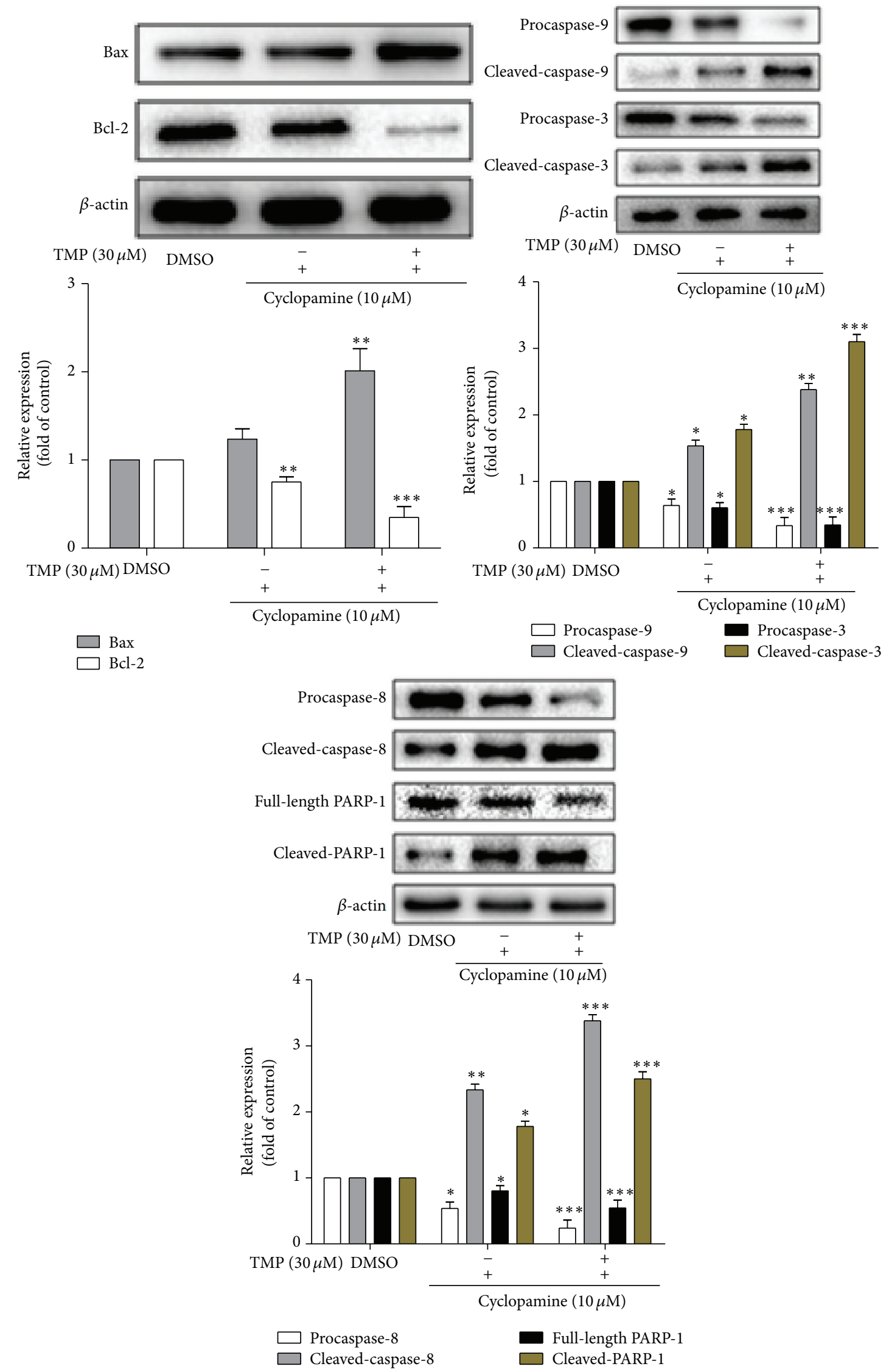

FIGURE 3: The effects of TMP on caspase activation and on the levels of Bcl-2/Bax proteins expressed in HSC-T6 cells. Data were reported as means \pm SD. For statistical analysis, ${ }^{*} p<0.05,{ }^{* *} p<0.01$, and ${ }^{* *} p<0.001$ represent significant difference. 


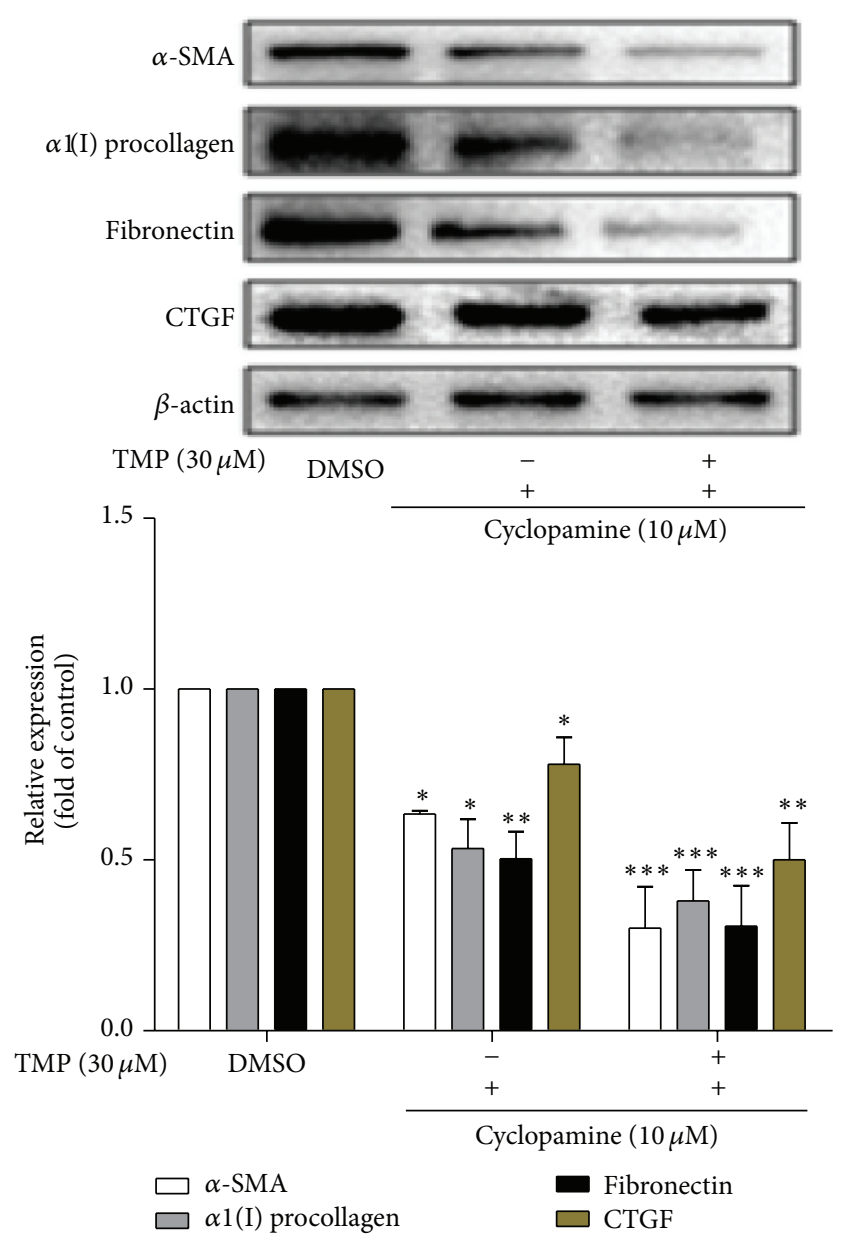

Figure 4: The effects of TMP on the levels of $\alpha$-SMA, $\alpha 1$ (I) collagen, fibronectin, and CTGF proteins expressed in HSC-T6 cells. Data were reported as means $\pm \mathrm{SD}$. For statistical analysis, ${ }^{*} p<0.05$, ${ }^{* *} p<0.01$, and ${ }^{* *} p<0.001$ represent significant difference.

that downstream target genes of the Hh signaling pathway were closely related to those of other signaling pathways, such as Wnt and TGF- $\beta$, which may be involved in the regulation of these signaling pathways [15]. Thus, investigation of TMP in regulating the Hh signaling pathway may establish a new approach for hepatic fibrosis treatment.

\section{Conflict of Interests}

The authors declare that there is no conflict of interests regarding the publication of this paper.

\section{Authors' Contribution}

Jue $\mathrm{Hu}$ and Gang Cao contributed equally to this work.

\section{References}

[1] S. K. Venkatesh, M. Yin, N. Takahashi, J. F. Glockner, J. A. Talwalkar, and R. L. Ehman, "Non-invasive detection of liver fibrosis: MR imaging features vs. MR elastography," Abdominal Imaging, vol. 40, pp. 766-775, 2015.

[2] B. Yilmaz and R. Dayanan, "Letter: reversibility of hepatic fibrosis and immunosuppression withdrawal in patients with autoimmune hepatitis/primary biliary cirrhosis overlap syndrome," Alimentary Pharmacology \& Therapeutics, vol. 41, no. 8, article 794, 2015.

[3] J.-J. Yang, H. Tao, and J. Li, "Hedgehog signaling pathway as key player in liver fibrosis: new insights and perspectives," Expert Opinion on Therapeutic Targets, vol. 18, no. 9, pp. 1011-1021, 2014.

[4] L. Hu, X. Lin, H. Lu, B. Chen, and Y. Bai, "An overview of hedgehog signaling in fibrosis," Molecular Pharmacology, vol. 87, no. 2, pp. 174-182, 2015.

[5] S. Wang, Y. Lee, J. Kim et al., "Potential role of hedgehog pathway in liver response to radiation," PLOS ONE, vol. 8, no. 9, Article ID e74141, 2013.

[6] J. Hyun, S. S. Choi, A. M. Diehl, and Y. Jung, "Potential role of Hedgehog signaling and microRNA-29 in liver fibrosis of IKK $\beta$ deficient mouse," Journal of Molecular Histology, vol. 45, no. 1, pp. 103-112, 2014.

[7] X. P. Zhang, F. Zhang, D. S. Kong et al., “Tetramethylpyrazine inhibits angiotensin II-induced activation of hepatic stellate cells associated with interference of platelet-derived growth factor beta receptor pathways," The FEBS Journal, vol. 281, no. 12, pp. 2754-2768, 2014.

[8] F. Zhang, Z. L. Zhang, D. S. Kong et al., “Tetramethylpyrazine reduces glucose and insulin-induced activation of hepatic stellate cells by inhibiting insulin receptor-mediated PI3K/AKT and ERK pathways," Molecular and Cellular Endocrinology, vol. 382, no. 1, pp. 197-204, 2014.

[9] F. Zhang, D.-S. Kong, Z.-L. Zhang et al., “Tetramethylpyrazine induces G0/G1 cell cycle arrest and stimulates mitochondrialmediated and caspase-dependent apoptosis through modulating ERK/p53 signaling in hepatic stellate cells in vitro," Apoptosis, vol. 18, no. 2, pp. 135-149, 2013.

[10] F. Zhang, C. Ni, D. Kong et al., "Ligustrazine attenuates oxidative stress-induced activation of hepatic stellate cells by interrupting platelet-derived growth factor- $\beta$ receptor-mediated ERK and p38 pathways," Toxicology and Applied Pharmacology, vol. 265, no. 1, pp. 51-60, 2012.

[11] G. Ji, L. Wang, S.-H. Zhang, J.-W. Liu, P.-Y. Zheng, and T. Liu, "Effect of Chinese medicine Qinggan Huoxuefang on inducing HSC apoptosis in alcoholic liver fibrosis rats," World Journal of Gastroenterology, vol. 12, no. 13, pp. 2047-2052, 2006.

[12] L. X. Tan, X. S. Li, Z. Q. Liu, and L. Y. Liu, "A randomized controlled study of ligustrazine in combination with propranolol for prevention of recurrent esophageal varices bleeding," Chinese Journal of Hepatology, vol. 8, no. 2, pp. 99-101, 2000.

[13] D. Camp, B. H. He, S. Li et al., "Ihog and Boi elicit Hh signaling via Ptc but do not aid Ptc in sequestering the Hh ligand," Development, vol. 141, no. 20, pp. 3879-3888, 2014.

[14] F. Mao, X. Yang, L. Fu et al., "The Kto-Skd complex can regulate ptc expression by interacting with Cubitus interruptus (Ci) in the hedgehog signaling pathway," Journal of Biological Chemistry, vol. 289, no. 32, pp. 22333-22341, 2014.

[15] F. Charron and M. Tessier-Lavigne, "The Hedgehog, TGF$\beta /$ BMP and Wnt families of morphogens in axon guidance," Advances in Experimental Medicine and Biology, vol. 621, pp. 116-133, 2007. 


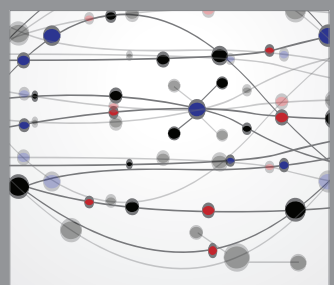

The Scientific World Journal
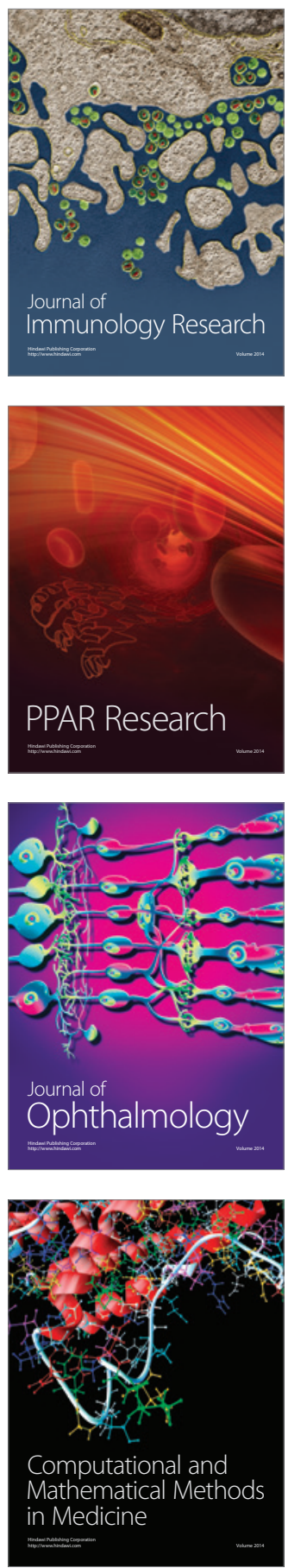

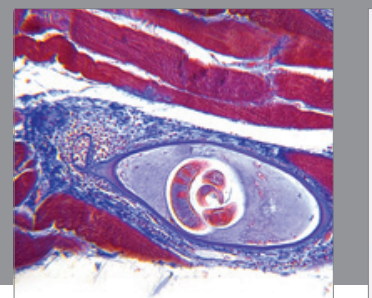

Gastroenterology

Research and Practice
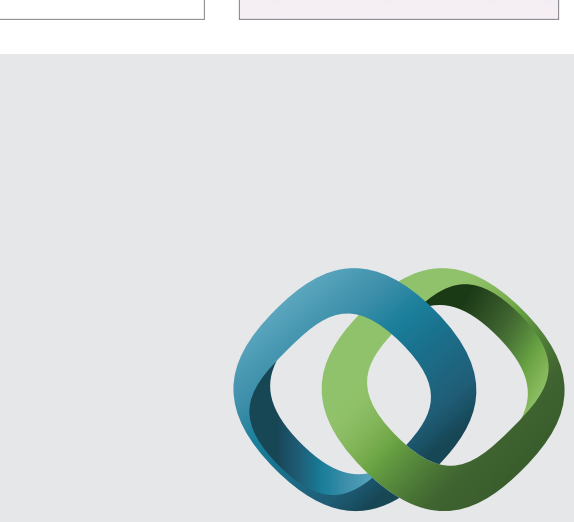

\section{Hindawi}

Submit your manuscripts at

http://www.hindawi.com
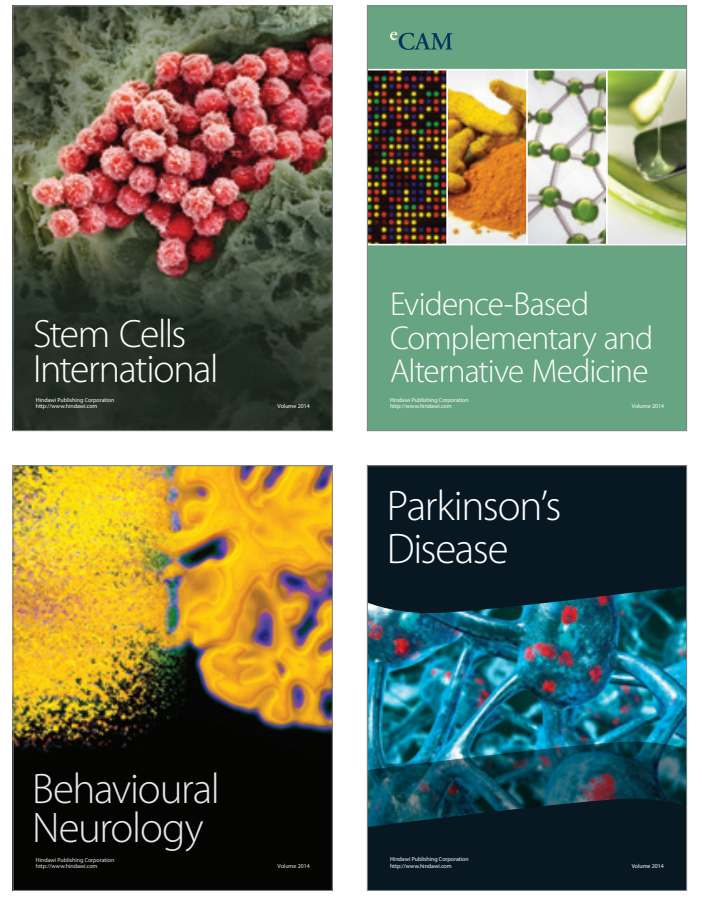
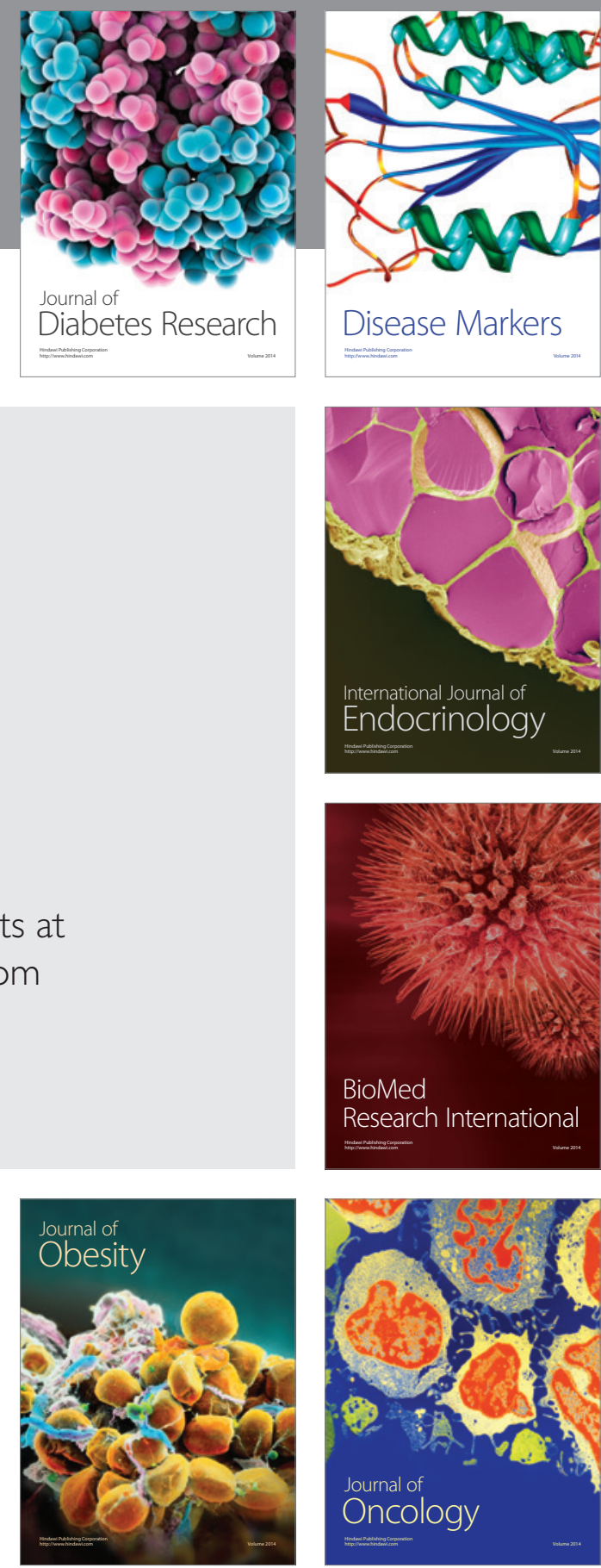

Disease Markers
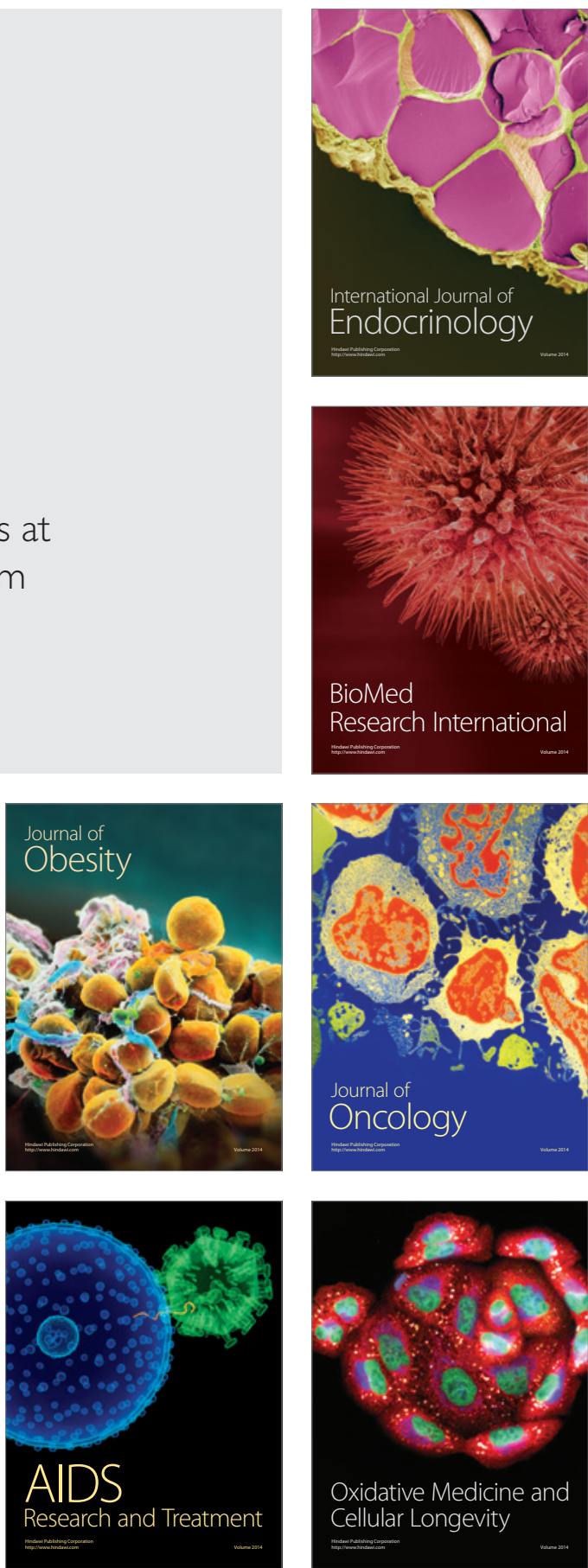